\title{
Compositional Averaging of Continuum Intensities in Multielement Compounds
}

\author{
John J. Donovan ${ }^{1, \star}$ and Nicholas E. Pingitore, $\mathrm{Jr}^{2}$ \\ ${ }^{1}$ Department of Earth and Planetary Science, The University of California, Berkeley, CA 94720-4767, USA \\ ${ }^{2}$ Department of Geological Sciences, The University of Texas at El Paso, El Paso, Texas 79968-0555, USA
}

\begin{abstract}
We find no physical basis for traditional mass-fraction weighted averaging of X-ray continuum measured in pure elements to estimate continuum production from compounds. Measurements of continuum from pairs of stable isotopes demonstrate that mass alone (the presence of more or fewer neutrons) has no effect on continuum production in electron probe microanalysis. Electron-fraction weighted averaging, which is based on the relative contribution of each constituent element to the electron (or proton) composition of a compound, proved superior to mass-fraction weighted averaging, in predicting the continuum of a compound from the measured continuum of its elements.
\end{abstract}

Key words: atomic fraction, atomic number averaging, average atomic number, bremsstrahlung, electron fraction, mass averaging, mass fraction, mean atomic number, X-ray continuum, z-bar

\section{INTRODUCTION}

Inelastic scattering is the primary mechanism for production of measurable X-ray continuum (bremsstrahlung). Calculations of X-ray continuum for multielement compounds traditionally have utilized mass-fraction averaging (Goldstein et al., 1992) of continuum measurements taken from pure elemental standards or proxies thereof. Nonetheless, fundamental physical considerations and isotope experiments (e.g., Donovan and Pingitore, 1998; Pingitore et al., $1999,2000)$ do not support the use of mass-based averaging for electron-solid interactions seemingly not related directly to mass. This paper deals with X-ray continuum production, a process that is distinct from characteristic $\mathrm{X}$-ray and backscattered electron productions.

Received April 23, 2001; accepted November 27, 2001.

*Corresponding author. E-mail: jdonovan@socrates.berkeley.edu
Does mass, in fact, have an unexpected effect on the production of X-ray continuum? X-ray continuum arises from the interaction of incident kinetic electrons with the Coulombic field of the atom. That field represents the total charges of the protons and electrons of the target, and is related to the number of each, that is, to $Z$. Therefore some variety of $Z$-based averaging should, in principle, be appropriate for continuum calculations in the multielement environment of compounds. Neutrons, possessing no electric charge, should have no measurable effect on the production of X-ray continuum at the electron-beam energies typical of electron probe microanalysis (EPMA). Yet conventional massfraction averaging is based on atomic weight, which is the sum of the masses of protons, electrons, and neutrons.

In an effort to detect an effect due to atomic weight, distinct from that due to atomic number, we performed precise measurements of X-ray continuum on pairs of different stable isotopes of individual elements. The only difference in such pairs is mass, the extra neutrons. If mass 
affects the production of X-ray continuum, then we expect to measure a difference in the continuum intensities of these stable isotope pairs. For this experiment, we compared X-ray continuum production in samples of normal $\mathrm{Cu}$ (average mass 63.54) and copper enriched in ${ }^{65} \mathrm{Cu}$; normal $\mathrm{Ni}$ (average mass 58.71) and nickel enriched in ${ }^{60} \mathrm{Ni}$; and normal Mo (average mass 95.94) and molybdenum enriched in ${ }^{100} \mathrm{Mo}$.

\section{Materials And Methods}

All measurements were performed on the Cameca SX-51 electron microprobe at the University of California at Berkeley, Department of Earth and Planetary Science. Experimental conditions for the X-ray continuum measurements were a beam of $100 \mathrm{nA}$ and $180 \mathrm{~s}$ counting time at $20 \mathrm{keV}$ and a beam of $150 \mathrm{nA}$ and $320 \mathrm{~s}$ counting time at $15 \mathrm{keV}$.

All acquisitions were performed at a dispersion crystal angle 0.01 sin theta above the actual line positions for $\mathrm{Na}$, $\mathrm{Al}, \mathrm{K}$, and $\mathrm{Fe} \mathrm{K} \alpha$ radiations which correspond to 12.1676, $8.5976,3.8289$, and $1.9776 \AA$ or $1.0190,1.4421,3.2382$, and $6.2696 \mathrm{keV}$. These positions were chosen to sample continuum at a range of energies.

WDS spectrometers were utilized rather than EDS spectrometers for two reasons: first, to achieve the desired statistics for continuum measurements, high beam currents were necessary. These would have had resulted in deadtimes close to $100 \%$ for EDS spectrometers due to the intensity of the characteristic lines that would also be present. Second, the high resolution of the WDS spectrometer greatly reduces the interferences from secondary characteristic lines that are very problematic at these precision levels.

The enriched isotopes were obtained from Oak Ridge National Laboratory with isotopic purities of 99.07\%, 99.7\%, and $94.5 \%$ for ${ }^{60} \mathrm{Ni},{ }^{65} \mathrm{Cu}$, and ${ }^{100} \mathrm{Mo}$, respectively. The elemental purity of our natural samples is $99.99 \%$ or better, and the elemental purities of the enriched isotopes are $\mathrm{Ni}$, 99.8\%; Cu, 99.98\%; and Mo, 99.99\%. Each natural or enriched isotope data point represents the average of 10-15 measurements.

Measurements of continuum on a variety of compounds, alloys, and pure elements were corrected for X-ray continuum absorption and anisotropy based on the method of Small et al. (1987), using mass absorption coefficients from McMaster et al. (1969), covering a range of average atomic number from 10 to 79 . Where error bars are not shown in the graphs of the results, one standard deviation is smaller than the symbol size.

\section{Results}

Figure $1(20 \mathrm{keV})$ and Figure $2(15 \mathrm{keV})$ both clearly demonstrate no significant difference between the continuum generated from the normal and from the isotopically enriched sample of each of the three elements tested (Ni, $\mathrm{Cu}$, and $\mathrm{Mo}$ ). Note that continuum measurements were taken at four different wavelengths, near the $\mathrm{K} \alpha$ peaks of $\mathrm{Na}, \mathrm{Al}, \mathrm{K}$, and $\mathrm{Fe}$. Continuum generated from the isotopic pairs of each element deviated less than $1 \%$ in the $20-\mathrm{keV}$ data, and less than $0.6 \%$ in the $15-\mathrm{keV}$ data, from the average of the two sets of measurements. Stated another way, the difference in the continuum generated from each isotopic composition of each element at each wavelength (a total of 12 tests at each accelerating potential) was less than $2 \%$ at $20 \mathrm{keV}$ and $1.2 \%$ at $15 \mathrm{keV}$. Significantly, in 10 of the 12 tests, the difference between the isotopic pair members was less than $1 \%$ for the $20-\mathrm{keV}$ data and in 9 of the 12 tests the difference between the isotopic pairs was less than $0.5 \%$ for the $15-\mathrm{keV}$ data.

The differences in the masses of the isotope pairs are $1.7 \%$ for $\mathrm{Ni}, 2.3 \%$ for $\mathrm{Cu}$, and $4 \%$ for Mo. The expected increase in continuum per atomic mass unit (under the assumption, with which we disagree, that continuum intensity is a function of mass) for pure elements in the region of $\mathrm{Ni}$ and $\mathrm{Cu}$ is approximately $1.9-2.3 \%$ for continuum energies around $\mathrm{Fe} \mathrm{K} \alpha$ and $\mathrm{K} \mathrm{K} \alpha$ and from $2.6-4.7 \%$ for energies around $\mathrm{Al} \mathrm{K} \alpha$ and $\mathrm{Na} \mathrm{K} \alpha$. This figure is based on the average changes in continuum production measured on pure $\mathrm{Si}, \mathrm{Ti}, \mathrm{V}, \mathrm{Co}, \mathrm{Cu}$, and $\mathrm{Ag}$, relative to the mass differences of these elements in their natural isotopic compositions. Likewise for the Mo region of the Periodic Table, continuum increases by about $0.3-1.3 \%$ per atomic mass unit. The expected differences in continuum production due to mass differences in our isotopic pairs thus are approximately $2-4 \%$ for $\mathrm{Ni}$ and $\mathrm{Cu}$ (depending on the continuum energy examined), and $1.2-5.3 \%$ for Mo (again varying with continuum energy). The data in Figures 1 and 2 thus present a strong case that mass does not contribute to continuum production.

Furthermore, there is no statistically significant pattern discernible that the heavier (or the lighter, for that matter) isotopic composition produces a higher intensity of continuum. For the $20-\mathrm{keV}$ data, only two cases show that the heavier composition produced more continuum and even then only by approximately one standard deviation, and in one case, the lighter yielded a greater intensity 

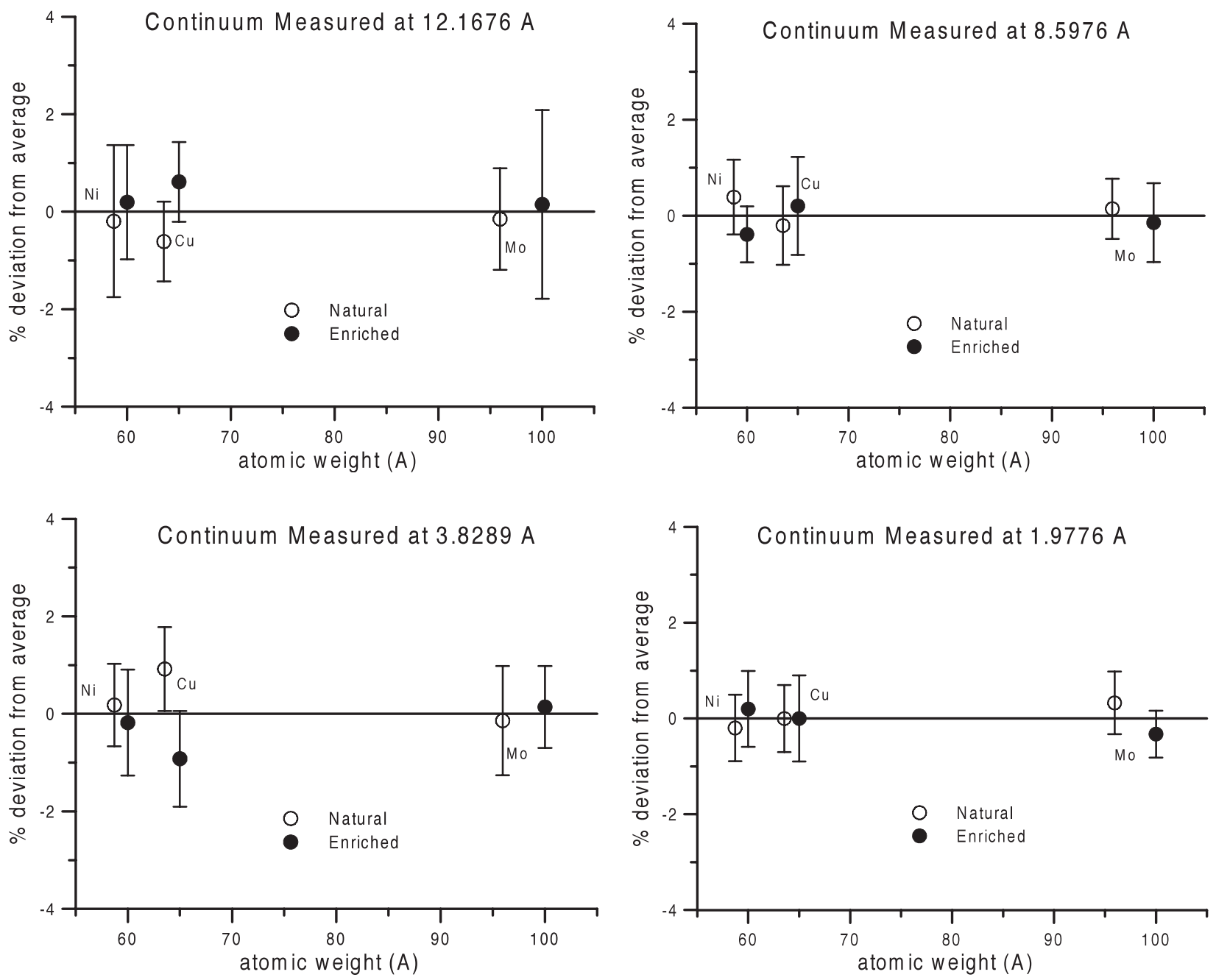

Figure 1. Comparison of X-ray continuum intensities for three stable isotope pairs. Data acquired at 0.01 sin theta above the actual line position for $\mathrm{Na} \mathrm{K} \alpha, \mathrm{Al} \mathrm{K} \alpha, \mathrm{K} \mathrm{K} \alpha$, and $\mathrm{Fe} \mathrm{K} \alpha$ (that is, precisely 12.1676, 8.5976, 3.8289, and $1.9776 \AA$ or $1.0190,1.4421,3.2382$, and $6.2696 \mathrm{keV}$, respectively). Note that the elements $\mathrm{Na}, \mathrm{Al}, \mathrm{K}$, and Fe were not present in our samples and their wavelengths served only as reference points for continuum measurements. Fractional atomic weights are averages for natural isotopic mixtures, presented for comparison with masses for enriched isotopes. Each point represents an average of 15 measurements $(20 \mathrm{keV}, 100 \mathrm{nA}, 180 \mathrm{~s}$ integration); each error bar is one standard deviation. Note that all the measurements fall within approximately $1 \%$ of the respective average of each isotope pair. These data indicate that mass, represented by excess neutrons, has no effect on continuum production.

also by approximately one standard deviation. In nine cases the yields appear statistically identical. For the $15-\mathrm{keV}$ data, there is an equal lack of pattern between the high and low atomic weight members of each isotope pair; in fact 9 out of 12 showed no statistically significant differences at all. Overall, the variation in the data seems random. Figures 1 and 2 thus force the conclusion that mass does not affect X-ray continuum production at the beam energies and precision levels typical of electron probe microanalysis.

\section{Discussion}

\section{Predicting Continuum of Compounds from Measured Continuum of Elements}

It is long established that atomic-fraction (mol-fraction) weighted averaging of the measured properties of the constituent pure elements poorly predicts the properties of compounds (Reed, 1993). Thus, a sample of uranium sul- 

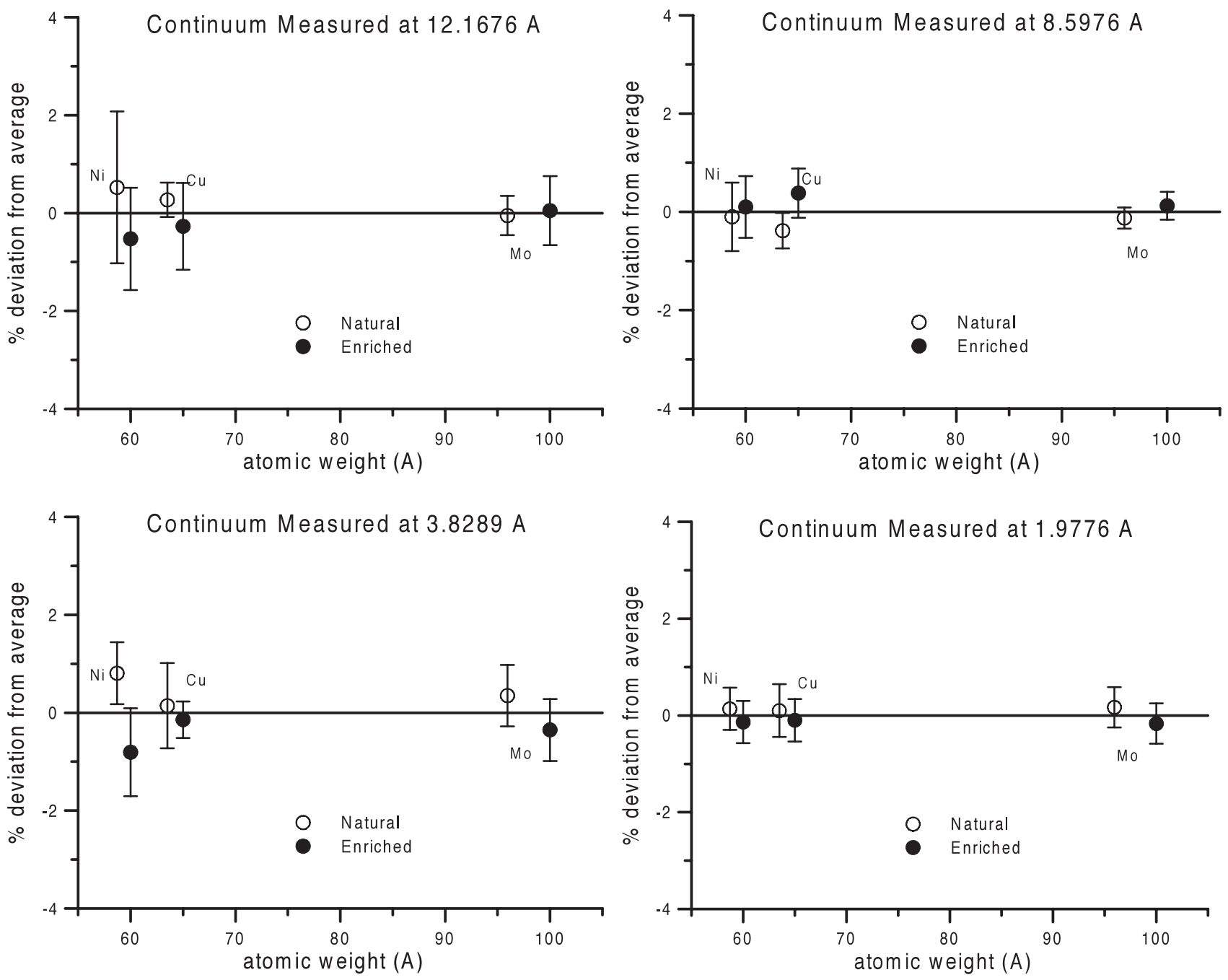

Figure 2. Comparison of X-ray continuum intensities for the three stable isotope pairs, at an accelerating voltage of $15 \mathrm{keV}$, and count time of $320 \mathrm{~s}$ at a current of $150 \mathrm{nA}$. Relative to the data in Figure 1, these data refer to continuum production at a lower accelerating potential. The increased beam currents and integration times result in further reduced statistical variation, and hence, no mass effect on continuum production is detectable approximately down to the $0.6 \%$ level. Each point represents an average of 12 measurements, and each error bar is again one standard deviation.

fide (US) generates intensities of such properties as continuum, characteristic X-rays, and backscattered electrons that are much closer to those of pure uranium than those of pure sulfur, even though the atomic proportion of the two elements is $1: 1$.

Pioneer electron microprobe scientists found empirically that mass-fraction weighted averaging provided reasonable estimates of the properties of compounds. Such massfraction weighted averaging became, and remains, an essential component of microprobe data manipulation. For the case of continuum production, Moreau and Calais (1964) and
Heinrich (1981) demonstrated a good fit between massfraction weighted averaging of continuum measurements taken on pure elements and continuum production in compounds.

Nonetheless, basic physics indicates that the kinetic electrons of the microprobe beam interact only with the electrons and protons of the target atoms, not with the neutrons. This consideration and measurements of characteristic X-ray generation from isotope pairs led us to develop electron-fraction weighted averaging (Donovan and Pingitore, 1998; Pingitore et al., 1999, 2000): 


$$
z_{i}=\frac{a_{i} Z_{i}}{\sum_{i=1}^{n} a_{i} Z_{i}}
$$

where $a_{i}$ is the atomic fraction and $Z_{i}$ is the atomic number of element $i$ in the compound. The difference between this expression and the expression for mass fraction is simply the substitution of atomic number for atomic weight. Because the ratio $A / Z$ is constant to a first approximation, mass-fraction and electron-fraction averaging yield results that generally are similar, but not identical.

Over the Periodic Table from Li to $\mathrm{U}$, the naturally occurring isotopic mixtures of some elements average more neutrons (and hence more mass) than might be expected from the $A / Z$ curve for all elements, while others have fewer neutrons (and hence less mass) than expected. There even are three places in the Periodic Table-Co-Ni, Ar-K, and $\mathrm{Te}-\mathrm{I}$ - where atomic mass decreases as atomic number increases. (This fact alone suggests concern for advocates of mass averaging of continuum and backscatter intensities.)

Since we have demonstrated that neutrons have no measurable effect on electron-solid interactions at typical EPMA energies and precision levels, mass-fraction averaging in traditional models imposes an error described by the variation of $A / Z$ with $Z$ for the naturally occurring elements. The magnitude of this error depends on the ratio of $A / Z$ for the elements of the compound in question. The difference between the mass fraction and electron fraction for the elements in many common compounds (e.g., minerals) is generally 1 to $3 \%$, but it can exceed $20 \%$ and $25 \%$ for such compounds as lead sulfide and uranium carbide (Table 1).

\section{Comparison of Mass-Fraction and Electron-Fraction Averaging}

The calculation of average atomic number by mass-fraction weighting for continuum intensities in a compound, $\bar{Z}$ (Z-bar), is given by (Goldstein et al., 1992)

$$
\bar{Z}_{\left(c_{i} Z_{i}\right)}=\sum_{i=1}^{n} c_{i} Z_{i}
$$

where $c_{i}$ is the mass fraction and $Z_{i}$ is the atomic number of element $i$ in the compound. We suggest that the electron fraction $\bar{Z}$ is calculated as (Pingitore et al., 1999)

$$
\bar{Z}_{\left(z_{i} Z_{i}\right)}=\sum_{i=1}^{n} z_{i} Z_{i}
$$

where $z_{i}$ is the electron fraction from equation (1).

\begin{tabular}{|c|c|c|c|c|}
\hline Compound & Element & $\begin{array}{l}\text { Mass } \\
\text { fraction }\end{array}$ & $\begin{array}{l}\text { Electron } \\
\text { fraction }\end{array}$ & $\begin{array}{r}\text { Relative } \\
\text { difference } \\
(\%)\end{array}$ \\
\hline \multirow[t]{2}{*}{$\mathrm{AuCu}$} & $\mathrm{Au}$ & 0.756 & 0.731 & -3.3 \\
\hline & $\mathrm{Cu}$ & 0.244 & 0.269 & 10.2 \\
\hline \multirow[t]{2}{*}{$\mathrm{PbS}$} & $\mathrm{Pb}$ & 0.866 & 0.837 & 20.4 \\
\hline & $S$ & 0.134 & 0.163 & 21.6 \\
\hline \multirow[t]{2}{*}{$\mathrm{NaCl}$} & $\mathrm{Na}$ & 0.393 & 0.393 & 0.0 \\
\hline & $\mathrm{Cl}$ & 0.607 & 0.607 & 0.0 \\
\hline \multirow[t]{2}{*}{ UN } & $\mathrm{U}$ & 0.944 & 0.929 & -1.6 \\
\hline & $\mathrm{N}$ & 0.056 & 0.071 & 26.7 \\
\hline \multirow[t]{2}{*}{$\mathrm{MgO}$} & $\mathrm{Mg}$ & 0.603 & 0.600 & -0.50 \\
\hline & $\mathrm{O}$ & 0.397 & 0.400 & 0.75 \\
\hline \multirow[t]{3}{*}{$\mathrm{ThSiO}_{4}$} & Th & 0.7159 & 0.6618 & -7.6 \\
\hline & $\mathrm{Si}$ & 0.0867 & 0.1029 & 18.6 \\
\hline & $\mathrm{O}$ & 0.1975 & 0.2353 & 19.1 \\
\hline \multirow[t]{2}{*}{$\mathrm{UC}_{2}$} & $\mathrm{U}$ & 0.983 & 0.8846 & -10.0 \\
\hline & $\mathrm{C}$ & 0.0917 & 0.1154 & 25.8 \\
\hline
\end{tabular}

Table 1. Range of Differences between Mass and Electron Fraction for a Variety of Compounds ${ }^{\mathrm{a}}$

aComparison of mass and "simple" electron fraction for a number of compounds. The relative difference between the two calculations depends on the $A / Z$ ratio of the elements in the compound and is due solely to variation in the ratio of neutrons to protons in the nucleus of the atom as a function of $Z$. Typically it is less than $5 \%$ but can be larger for certain compounds such as $\mathrm{PbSiO}_{3}$ (Alamosite) and can even exceed 20 or $25 \%$ relative for compounds which contain elements with extremely disparate $A / Z$ ratios.

Figure 3 plots measured X-ray continuum of compounds against average atomic number of the compounds calculated by mass-fraction (Fig. 3a) and electron-fraction (Fig. 3b) weighting, along with a second-order polynomial fit to the continuum data in the $\mathrm{Fe} \mathrm{K} \alpha$ region. Identical results were obtained for all expressions in the softer X-ray continuum regions, although the increased incidence of higher-order WDS interferences at these longer wavelengths renders the data analysis more problematic.

Results from mass and electron fraction expressions are similar, although the electron-fraction average produces slightly better fit statistics: residual sum of squares $=568.0$, coefficient of determination ( $r$-squared) $=0.9941$ for electron fraction averaging compared to residual sum of squares $=808.4$, coefficient of determination $(r$-squared $)=$ 0.9917 for mass fraction averaging. 
a.

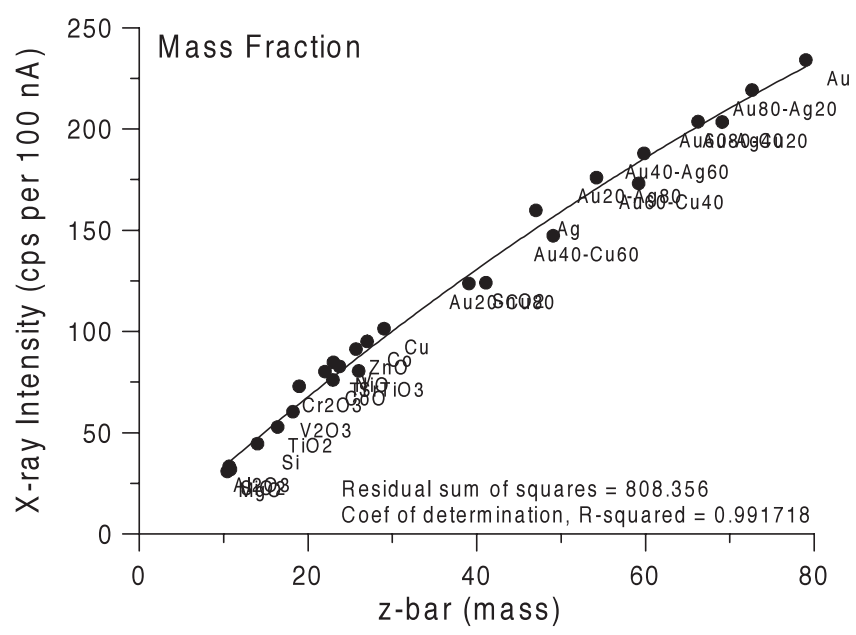

c.

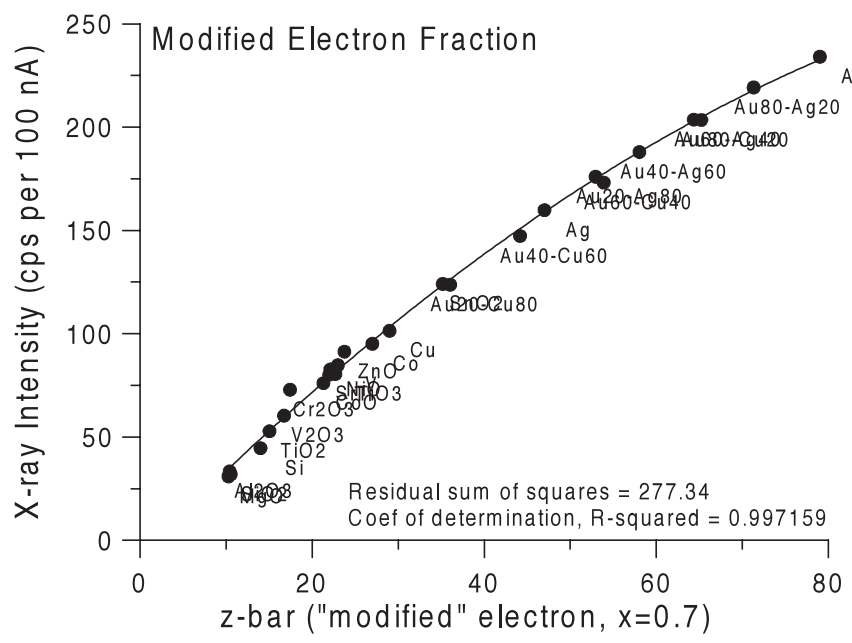

b.

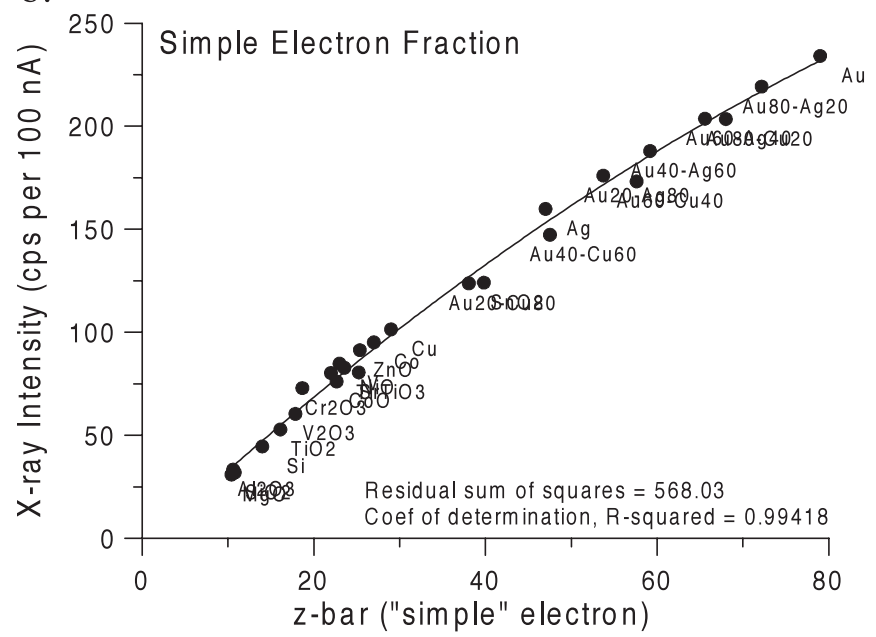

d.

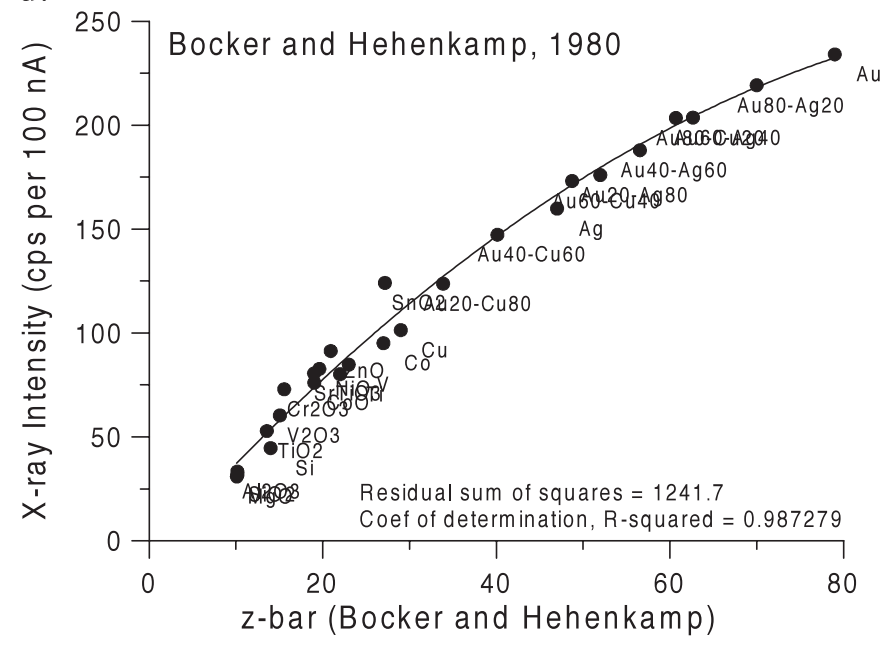

e.

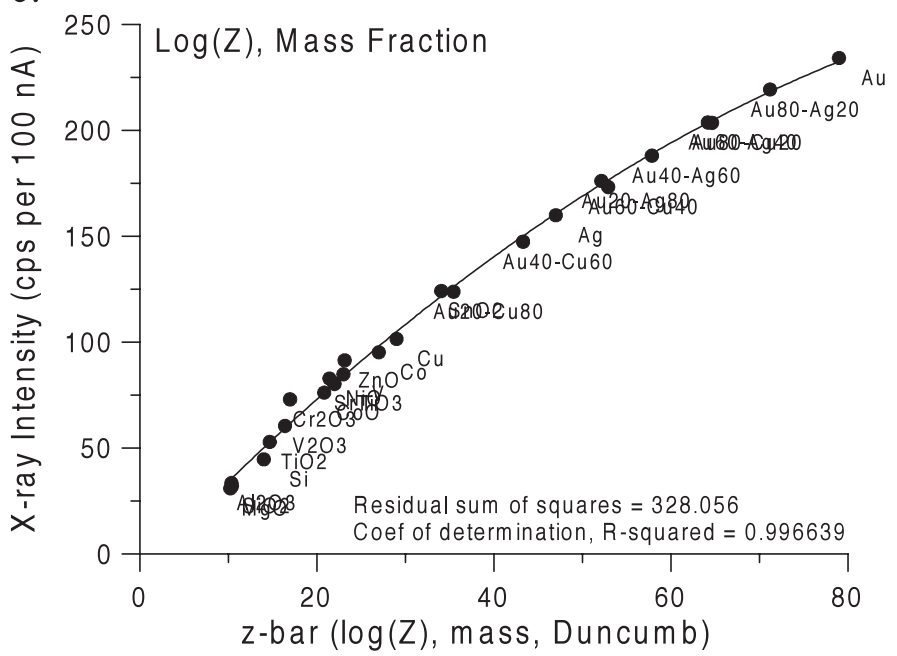

Figure 3. (See caption on facing page.) 


\section{Modified Averaging to Achieve Better Predictions}

Both the mass-fraction and electron-fraction weighted averaging schemes, equations (2) and (3), represent linear mixing. Because there is no physical constraint that demands linear mixing of continuum production, we present a simple nonlinear electron-fraction weighted averaging, and compare it with two nonlinear mass-fraction weighted averaging schemes for X-ray continuum, one proposed by Bocker and HehenKamp (1980) and another provided by Peter Duncumb of Cambridge (unpublished).

We added an exponent, $x$, to create $Z_{i}^{x}$ in the expression for electron fraction, yielding the following:

$$
z_{i}^{(x)}=\frac{a_{i} Z_{i}^{x}}{\sum_{i=1}^{n} a_{i} Z_{i}^{x}} .
$$

Therefore, by substituting this modified electron fraction, $z_{i}^{(x)}$, in equation (4) for $z_{i}$ in equation (3), we obtain

$$
\bar{Z}_{\left(z_{i}^{(x)} Z_{i}\right)}=\sum_{i=1}^{n} z_{i}^{(x)} Z_{i} .
$$

We then varied the value of $x$ and by trial and error, found that an exponent of $x=0.7$ in equation (4) noticeably improved the fit for X-ray continuum plotted as a function of $\bar{Z}$. Figure $3 \mathrm{c}$ presents the results from this modified, nonlinear electron-fraction model; compare with Figure $3 \mathrm{~b}$.

Bocker and HehenKamp (1980) suggested the following modification of traditional mass-fraction weighted average:

$$
\bar{Z}_{\left(c_{i} Z_{i}^{0.25}\right)}=\sum_{i=1}^{n} c_{i} Z_{i}^{0.25} .
$$

The modified electron-fraction fit, Figure $2 c$, is seen to be superior to that obtained in Figure $3 d$ using the Bocker and HehenKamp (1980) modified mass-fraction fit, which was optimized for continuum measurements in the $\mathrm{Au}-\mathrm{Cu}-\mathrm{Ag}$ system only.

A better fit to the data for mass-fraction weighted averaging is obtained from the unpublished expression provided by Duncumb:

$$
\bar{Z}_{\left(c_{i} \ln Z_{i}\right)}=e^{\sum_{i=1}^{n} c_{i} \ln Z_{i}},
$$

where the $\bar{Z}$ is calculated from the mass fraction sum of the natural $\log$ of $Z$ for each element. The Duncumb fit, as seen in Figure $3 \mathrm{e}$, is superior to both the traditional and the Bocker and HehenKamp (1980) mass-fraction weighted fits.

Nonetheless, the electron-fraction weighted fit is still statistically slightly better than the new Duncumb fit: residual sum of squares $=277.3$, coefficient of determination $(r$-squared $)=0.9972$ for the modified nonlinear electronfraction averaging compared to residual sum of squares $=$ 328.0 , coefficient of determination $(r$-squared $)=0.9966$ for $\log Z$ mass-fraction averaging.

\section{CONCLUSIONS}

On the basis of fundamental physics and the results of our isotope experiments, we conclude that mass alone (in contrast to electric charge) has no measurable effect on continuum production in electron microprobe analysis. It is thus inappropriate to apply mass-fraction weighted averaging of continuum intensities measured on pure elements to estimate continuum production of compounds.

Electron-fraction weighted averaging is a physically realistic approach to prediction of continuum production in compounds. We demonstrate that electron-fraction weighted

Figure 3. Measurements of X-ray continuum for various compounds, alloys, and pure elements, plotted against average atomic number, as calculated from (a) mass fraction; (b) electron fraction; (c) modified electron fraction, equation (5); (d) modified mass fraction, Bocker and HehenKamp (1980); and (e) modified mass fraction, Duncumb (unpublished). Continuum intensity given in cps per $100 \mathrm{nA}$, taken at $20 \mathrm{keV}, 180 \mathrm{~s}$, average of 10-15 points, acquired at 0.01 sin theta above the actual line position for $\mathrm{Fe} \mathrm{K} \alpha$. All X-ray continuum data were corrected for continuum absorption and anisotropy using the technique of Small et al. (1987) and mass absorption coefficients of McMaster et al. (1969). Electron-fraction averaging (b) yielded better fit than mass-fraction averaging (a). For nonlinear mixing models, modified electron fraction averaging (c) was superior to modified mass-fraction averaging (d, e). 
averaging provides a fit to the experimental data that is clearly superior to that from mass-fraction weighted averaging, especially for those compounds containing elements that exhibit large differences in $A / Z$ ratios.

In an effort to improve the predictive power of the electron-fraction weighted averaging, we turned to a simple nonlinear mixing model. This slightly but noticeably improved the estimates for continuum production in compounds and was superior to two previously proposed nonlinear models based on mass-fraction averaging.

\section{ACKNOWLEDGMENTS}

We thank Andrew Westphal (UC Berkeley) for helpful criticism and Peter Duncumb (Cambridge) for suggesting equation (7). JJD also thanks the Department of Earth and Planetary Science at UC Berkeley for financial support and Tim Teague for meticulous sample preparation.

\section{REFERENCES}

Bocker, J. \& HehenKamp, T. (1980). Continuum-radiation in quantitative electron microprobe analysis. In 8th International Congress of X-ray Optics and Microanalysis, D.R. Beaman, R.E.
Ogilvie, \& D.B. Wittry (Eds.), pp. 77-86. Midland, Michigan: Pendell Publishing Co.

Donovan, J.J. \& Pingitore, N.E., JR. (1998). The effect of mass in electron-solid interactions and the mystery of the "Heinrich kink." Microsc Microanal 4(Suppl. 2), 250-251.

Goldstein, J.I., Newbury, D.E., Echlin, P., Joy, D.C., Romig, A.D., Lyman, C.E., Fiori, C. \& Lifshin, E. (Eds.). (1992). Scanning Electron Microscopy and X-ray Microanalysis. 2nd ed. New York and London: Plenum Press.

Heinrich, K. (1981). Electron Beam X-Ray Microanalysis. New York: Van Nostrand Reinhold.

McMaster, W.H., Kerr Del Grande, N., Mallet, J.H. \& HubBeLL, J.H. (1969). Compilation of X-ray Cross Sections. Livermore, California: Lawrence Livermore Laboratory Press.

Moreau, G. \& Calais, D. (1964). Determination du numero atomique moyen d'un binaire homogen $\mathrm{AB}$ (solution solide du compose defini). J Phys 25(Suppl 6), 83.

Pingitore, N.E., Donovan, J.J. \& Jeanloz, R. (1999). Electron microprobe quantification: A new model based on electrons rather than on mass. J Appl Phys 86, 2790-2794.

Pingitore, N.E., Donovan, J.J. \& Jeanloz, R. (2000). Response to comment on "Electron microprobe quantification: A new model based on electrons rather than on mass." J Appl Phys 87, 8221.

Reed, S.J.B. (1993). Electron Microprobe Analysis, 2nd Ed., Cambridge: Cambridge University Press.

Small, J.A., Leigh, S.D., Newbury, D.E. \& Myklebust, R.L. (1987). Modeling of the bremsstrahlung radiation produced in pure element targets by $10-40 \mathrm{keV}$ electrons. J Appl Phys 61(2), 459-469. 